\section{CACN \\ Platform Presentations}

\section{D.01}

\section{Video diversion improves success rate of fundoscopic exam in children: a randomized controlled trial}

MM Yang (Calgary)*A Singhal (Vancouver) JM Kerr (Vancouver) AR Hengel (Vancouver) S George (Vancouver) P Thiessen (Vancouver) K Muir (Vancouver) P Enarson (Vancouver)

doi: $10.1017 / \operatorname{cjn} .2015 .82$

Background: Fundoscopy is an important component of the neurological examination, but can be challenging in uncooperative children. This study explores whether playing a video during eye examination, improves the success, duration and ease of pediatric fundoscopy. Methods: We completed a prospective, multi-clinic, block-randomized trial. Patients 1-4 years were recruited in the emergency department, neurology, spinal cord and general pediatric clinic. Patients were randomized (by eye examined) to video/nonvideo assisted fundoscopy. Successful exams were defined as visualizing the fundus within 60 seconds. Time to visualize optic disc was recorded and difficulty of exam was examined using a 10-point Likert scale. Results: 101 subjects were recruited, with a mean age of 2.8 years. Overall, there was a $20 \%$ absolute improvement in the success rate of visualizing the optic disc in the video versus non-video group $(\mathrm{p}<0.01)$. Time to visualize optic disc was also improved $(\Delta 5.3 \mathrm{~s}$, $\mathrm{p}<0.01)$. Improvement in ease of examination with video were noted by caregivers and practitioners $(\mathrm{p}<0.01)$. Conclusion: Playing a video improved the ease, duration and most importantly the success of fundoscopy in younger children. This simple, inexpensive adjunct has great potential to improve the ease and efficacy of this aspect of the neurological examination.

\section{D.02}

2015 update on triple therapy for pyridoxine-dependent epilepsy

S Jaggumantri (Vancouver) C Coughlin (Aurora) S Stockler (Vancouver) C Barb (Vancouver) C Mary (Vancouver) J van Hove (Aurora) C van Karnebeek (Vancouver)*

doi: 10.1017/cjn.2015.83

Background \& Objectives: Of patients with pyridoxinedependent epilepsy (PDE) due to ATQ deficiency, 75\% suffer intellectual disability despite adequate seizure control with high dose pyridoxine. We aimed to assess the safety and efficacy of two novel therapeutic strategies to reduce accumulation of toxic intermediates in this cerebral lysine degradation defect. Methods: In two open-label observational studies, seven children with confirmed ATQ deficiency were started on dietary lysine restriction with regular nutritional monitoring, and outcome evaluation pipecolic acid, AASA levels in body fluids; development/cognition via age-appropriate tests and parental observations; epilepsy). Subsequently additional arginine supplementation was initiated to reduce cerebral lysine flux (cation transporter competitive inhibition).. Results: Lysine-restriction was well tolerated and diet is safe, resulted in reduction of lysine intermediates in all body fluids in all patients (up to $80 \%$ reduction AASA in cerebrospinal fluid), with beneficial effects on seizure control and psychomotor development. Additional arginine fortification resulted in normalization of biomarkers and dramatic improvement of psychomotor development. Discussion: Triple therapy is effective, especially if implemented early; studies for PDE newborn screening have been initiated. For dissemination and evidence generation, our PDE Consortium published Recommendations, developed a Digital Diet App and established a RedCap study database (www.pdeonline.org).

\section{D.03}

Canadian Paediatric Neurology Workforce survey and consensus statement

A Doja (Ottawa) S Orr (Ottawa) H McMillan (Ottawa) A Kirton (Calgary)*D Callen (Hamilton)

doi: 10.1017/cjn.2015.84

Background: Little knowledge exists on the availability of paediatric neurology positions. Our study aimed to: 1) obtain information regarding the availability of positions for paediatric neurologists in academic centres; 2) survey paediatric neurology trainees regarding their perceptions of employment issues and career plans; 3 ) survey practicing community paediatric neurologists 4) convene a group of paediatric neurologists to develop a consensus regarding how to address these workforce issues. Methods: Surveys addressing workforce issues regarding paediatric neurology in Canada were sent to: 1) all paediatric neurology program directors in Canada who then solicited information from division heads and from paediatric neurologists in surrounding areas; 2) paediatric neurology trainees in Canada and; 3 ) community paediatric neurologists. A meeting was held with relevant stakeholders to develop a consensus on how to approach employment issues. Results: We found that the number of projected positions in academic paediatric neurology is fewer than the number of paediatric neurologists that are being trained. Paediatric neurology residents are concerned about job availability and desire more career counselling. Conclusions: There is a current and projected clinical demand for paediatric neurologists despite a lack of academic positions. Training programs should focus on community neurology as a viable career option.

\section{D.04}

Interplay between impaired brain structure and function in term newborns with congenital heart disease

A Birca (Toronto)* VA Vakorin (Toronto) S Madathil (Toronto) V Chau (Toronto) SP Miller (Toronto) SM Doesburg (Toronto) M Seed (Toronto) S Blaser (Toronto) DA Nita (Toronto) EG Duerden (Toronto) EJ Hickey (Toronto) CD Hahn (Toronto)

doi: $10.1017 /$ cjn.2015.85

Background: Term neonates with congenital heart disease (CHD) demonstrate a high incidence of white matter injury (WMI), together with increased average diffusivity and decreased fractional anisotropy (FA) on MR diffusion tensor imaging. EEG background activity is a robust measure of functional brain maturation that becomes less discontinuous, contains more fast activity and shows higher complexity of EEG patterns with increasing age. We sought to determine the association between structural brain abnormalities and functional brain maturation in term neonates with CHD. Methods: 